\title{
Corilagin induces the apoptosis of hepatocellular carcinoma cells through the mitochondrial apoptotic and death receptor pathways
}

\author{
YUAN DENG ${ }^{1,2^{*}}$, XUDAN $\mathrm{LI}^{1,2^{*}}, \mathrm{XUAN}^{\mathrm{I}}{ }^{1,2}, \mathrm{ZHIZHONG} \mathrm{ZHENG}^{1,3}$, WEN HUANG $^{3}$, \\ LIANGHUA CHEN $^{3}$, QINGXUAN TONG ${ }^{3}$ and YANLIN MING ${ }^{1-3}$
}

\author{
${ }^{1}$ Key Laboratory of Xiamen City for Plant Introduction and Quarantine and Plant Products, \\ Xiamen Overseas Chinese Subtropical Plant Introduction Garden, Xiamen, Fujian 361002; \\ ${ }^{2}$ Department of Bioengineering and Biotechnology, College of Chemical Engineering, Huaqiao University, \\ Xiamen, Fujian 361021; ${ }^{3}$ Key Laboratory of Fujian Province for Physiology and Biochemistry of Subtropical Plant, \\ Fujian Institute of Subtropical Botany, Xiamen, Fujian 361006, P.R. China
}

Received October 29, 2017; Accepted March 29, 2018

DOI: 10.3892/or.2018.6396

\begin{abstract}
Corilagin, a gallotannin, is one of the major active components of many ethnopharmacological plants and exhibits antitumor, anti-inflammatory and antioxidative properties. In recent years, corilagin has provoked much attention due to its antitumor activity, yet the mechanisms attributed to its anticancer actions are largely unknown. In our previous research, our group reported that corilagin could inhibit the proliferation of hepatocellular carcinoma (HCC) cells by inducing $\mathrm{G} 2 / \mathrm{M}$ phase arrest. In the present study, observation of the morphological changes showed that corilagin induced the apoptosis of HCC cells as determined by AO/EB and Hoechst 33258 staining assays. Furthermore, flow cytometric analysis was carried out to calculate the apoptotic rate which was $24.1 \%$ following treatment with corilagin $(37.5 \mu \mathrm{M})$. At the molecular level, mitochondrial membrane potential assay and western blot analysis showed that the mitochondrial transmembrane potential was reduced and the rate of release
\end{abstract}

Correspondence to: Dr Yanlin Ming, Key Laboratory of Xiamen City for Plant Introduction and Quarantine and Plant Products, Xiamen Overseas Chinese Subtropical Plant Introduction Garden, Xiamen, Fujian 361002, P.R. China

E-mail: xmyanlin@gmail.com

*Contributed equally

Abbreviations: P., Phyllanthus; HCC, hepatocellular carcinoma; HBV, hepatitis B virus; CDDP, cis-diamminedichloroplatinum (cisplatin); $\mathrm{IC}_{50}, 50 \%$ inhibitory concentration; PI, propidium iodide; PARP, poly(ADP-ribose) polymerase; $\mathrm{AO} / \mathrm{EB}$, acridine orange/ethidium bromide; MTT, 3-(4,5-dimethylthiazol-2-yl)-2,5-diphenyltetrazolium bromide (methyl thiazolyl tetrazolium); PMSF, phenylmethylsulfonyl fluoride

Key words: corilagin, hepatocellular carcinoma, apoptosis, signaling pathway of cytochrome $c$ was increased, which led to the activation of caspase-9, caspase-3 and cleavage of PARP in the cytoplasm indicating activation of the mitochondrial apoptotic pathway. Moreover, following treatment with corilagin, we noted upregulation of Fas and FasL and activation of caspase-8 which represented activation of the death receptor pathway, and we also observed downregulation of $\mathrm{Bcl}-2$ and survivin which was also attributed to the antitumor effect of corilagin. These results suggest that corilagin significantly induced the apoptosis of HCC cells through both the mitochondrial apoptotic pathway and the death receptor pathway, and corilagin is a potential complementary anticancer herbal drug for HCC therapy.

\section{Introduction}

Hepatocellular carcinoma (HCC) is the third leading cause of cancer-related death cancer worldwide. The incidence and mortality of HCC are increasing in Asian countries as a result of an ageing cohort infected with hepatitis B virus (HBV), and these parameters are expected to continue to rise as a consequence of the obesity epidemic or exposure to a contaminated environment (1). Most HCC patients typically present in advanced and incurable stages, and are usually unsuitable for surgery. However, this situation is improving with a better understanding of the molecular mechanisms involved in the progression of HCC (2). Recently, researchers have focused on plant-derived compounds that have the potential to target the molecules which are critical for the progression of HCC (3).

Corilagin (Fig. 1), a natural plant polyphenol tannic acid, is a major active component of many ethnopharmacological plants such as Phyllanthus $(P$.) niruri L., P. emblica L. and $P$. urinaria L. It was first isolated from Caesalpinia coriaria (Jacq.) Willd. (Divi-divi) by Schmidt in 1951 (4). In recent years, corilagin has provoked much attention due to its antitumor, hepatoprotective, and anti-inflammatory activities, but particularly as a candidate antitumor agent. In the past few decades, corilagin has been reported to display various pharmacological activities, including antioxidant activity (5), hepatoprotective effect (6), anti-inflammatory activity (7), 
neuroprotective effect (8), cardiovascular protective activity (9) and anti-diabetic activity (10). Recently, the antitumor effect of corilagin $(2,3,11,12)$ has been a focus of increased research.

Research on the antitumor activity of corilagin began in 1985. Corilagin was reported to inhibit the reverse transcriptase activity of RNA tumor virus (13), to inhibit the topoisomerase I-mediated relaxation of DNA, which inhibits the growth of tumor cells $(14,15)$, and to reduce the release of tumor necrosis factor- $\alpha$ (TNF- $\alpha$ ) in cancer cells. TNF- $\alpha$ is thought to stimulate the growth and progression of early malignant tumor cells $(16,17)$. In recent years, corilagin has been demonstrated to suppress human colon cancer, gastric adenocarcinoma and metrocarcinoma (18-20), to inhibit the growth of tumor cells in vivo, to suppress the proliferation of HCC Hep3B cells in an athymic nude mouse xenograft model (11), to suppress the growth of HCC cells by inducing G2/M phase arrest and to inhibit the proliferation of ovarian cancer cells via the TGF- $\beta /$ AKT/ERK signaling pathways $(2,3)$. In addition, corilagin was found to inhibit the proliferation of cholangiocarcinoma (CCA) cells by regulating the Notch signaling pathway. In vitro, corilagin inhibited CCA cell proliferation, migration and invasion, and induced the apoptosis of CCA cells by inhibiting the Notch signaling pathway. Moreover, corilagin has shown the potential to promote the antitumor activity of cisplatin and doxorubicin in HCC cells $(11,21)$ and to sensitize ovarian cancer cells to paclitaxel and carboplatin by inhibiting the Snail-glycolysis pathways (22).

Based on the above findings, corilagin induces the apoptosis of HCC cells, yet the mechanism remains unclear. Therefore, the present study aimed to explore the molecular mechanisms involved in the pro-apoptotic effect of corilagin and to provide evidence that corilagin may have the potential to become a complementary anticancer herbal drug for HCC therapy.

\section{Materials and methods}

Chemicals and reagents. Corilagin (purity $\geq 98 \%$ ) was prepared at the Xiamen Overseas Chinese Subtropical Plant Introduction Garden (Xiamen, China) as previously described (21). Three HCC cell lines (SMMC-7721, Bel-17402 and MHCC97-H) were used and all were obtained from the Cell Bank of the Chinese Academy of Sciences (Shanghai, China). RPMI-1640 medium and Dulbecco's modified Eagle's medium (DMEM) were obtained from Gibco (Thermo Fisher Scientific, Inc., Waltham, MA, USA). Bisbenzimide (Hoechst 33258), acridine orange (AO), JC-1, ethidium bromide (EB), methyl thiazolyl tetrazolium (MTT) and cisplatin (CDDP; purity $\geq 99.9 \%$ ) were obtained from Sigma-Aldrich (Merck KGaA, Darmstadt, Germany). Annexin V-FITC/PI Apoptosis Detection kit was obtained from Roche (Mannheim, Germany). The antibodies against p53 (1:1,000; mouse mAb; cat. no. 48818), Bcl-2 (1:1,000; mouse mAb; cat. no. 15071), cytochrome $c(1: 1,000$; rabbit mAb; cat. no. 11940), caspase-9 (1:1,000; mouse mAb; cat. no. 9508), caspase-8 (1:1,000; mouse mAb; cat. no. 9746), caspase-3 (1:1,000; rabbit mAb; cat. no. 9662), PARP (1:1,000; rabbit $\mathrm{mAb}$; cat. no. 9746), surviving (1:1,000; mouse $\mathrm{mAb}$, cat. no. 2802), Fas (1:1,000; mouse mAb; cat. no. 8023) and FasL (1:1,000; rabbit mAb; cat. no. 4273) were purchased from Cell Signaling Technology (Danvers, MA, USA).

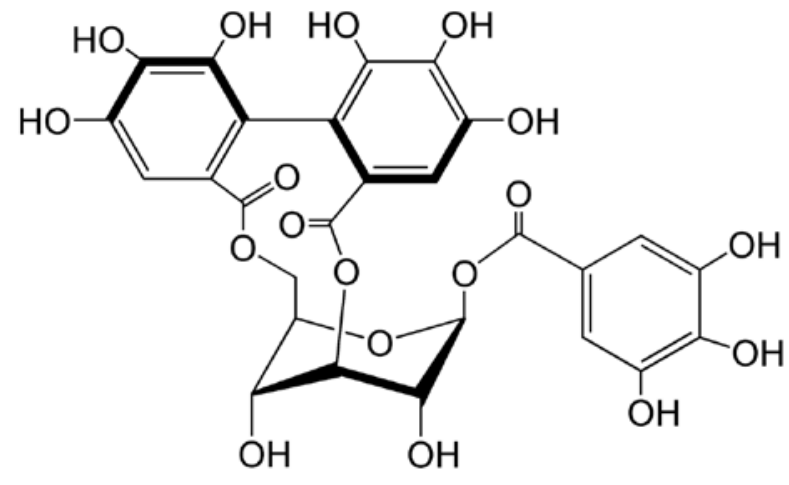

Figure 1. The chemical structure of corilagin $\left(\mathrm{C}_{27} \mathrm{H}_{22} \mathrm{O}_{18}\right)$.

Cell culture. Cells were cultured in RPMI-1640 or DMEM supplemented with $10 \%$ FBS, penicillin $(100 \mathrm{U} / \mathrm{ml})$ and streptomycin $(100 \mathrm{mg} / \mathrm{ml})$ in a humidified incubator aerated with $5 \% \mathrm{CO}_{2}$ and $95 \%$ air at $37^{\circ} \mathrm{C}$. When the cells reached $70-80 \%$ confluency, they were trypsinized and counted, and 5,000 cells were seeded into each 96 -well microtiter plate for overnight incubation and then treated with corilagin complete cell medium for $48 \mathrm{~h}$. The control group was treated with DMSO $(0.1 \%, \mathrm{w} / \mathrm{v})$ for the same duration.

MTT assay. The effect of the constituents on the growth of the HCC cells was determined using the MTT assay. Briefly, the cells were seeded overnight and the medium was removed. The cells were then treated with $180 \mathrm{ml}$ corilagin dissolved in medium at $6.25,12.5,25,50,75$ and $100 \mu \mathrm{M}$ or with DMSO $(0.1 \%, w / v)$ as a control for $24 \mathrm{~h}$. After $24 \mathrm{~h}, 20 \mu \mathrm{l}$ MTT $(5 \mathrm{mg} / \mathrm{ml})$ was added to each well, and the plates were incubated for an additional $4 \mathrm{~h}$ at $37^{\circ} \mathrm{C}$. The supernatants were replaced with $150 \mathrm{ml}$ of DMSO to dissolve the formazan produced from the MTT by the metabolizing cells. Absorbance at $492 \mathrm{~nm}$ was proportional to the live metabolizing cell count, and cell survival was expressed as the absorbance of the MTT-treated cells relative to that of the DMSO-treated controls (absorbance was detected using Thermo Scientific Microplate Reader MK3).

Colony formation assay. Cells were seeded at 800 cells per well in a 6-well plate $\left(9.5 \mathrm{~cm}^{2}\right)$ and allowed to attach overnight prior to treatment with DMSO or varying concentrations of corilagin. The cells were treated for $24 \mathrm{~h}$, then recovery medium was added and the cells were allowed to continue to grow for 10 days. Colonies were stained using $0.5 \%$ crystal violet solution in methanol for $30 \mathrm{~min}$. Staining solution was removed, wells were washed with deionized $\mathrm{H}_{2} \mathrm{O}$, and the stained colonies containing more than 50 cells were imaged and counted under microscopy (Leica Microsystems GmbH, Wetzlar, Germany).

Morphological studies using fluorescence microscopy. The HCC cells seeded into a 6-well plate were treated with $37.5 \mu \mathrm{M}$ corilagin or DMSO $(0.1 \%, \mathrm{w} / \mathrm{v})$ as a negative control and $30 \mu \mathrm{M}$ $\mathrm{CDDP}$ as a positive control for $24 \mathrm{~h}$. The supernatants were then replaced by PBS containing $100 \mu \mathrm{g} / \mathrm{ml}$ of Hoechst 33258 or AO/EB in PBS for 10 min in the dark. Cell morphology was 
A
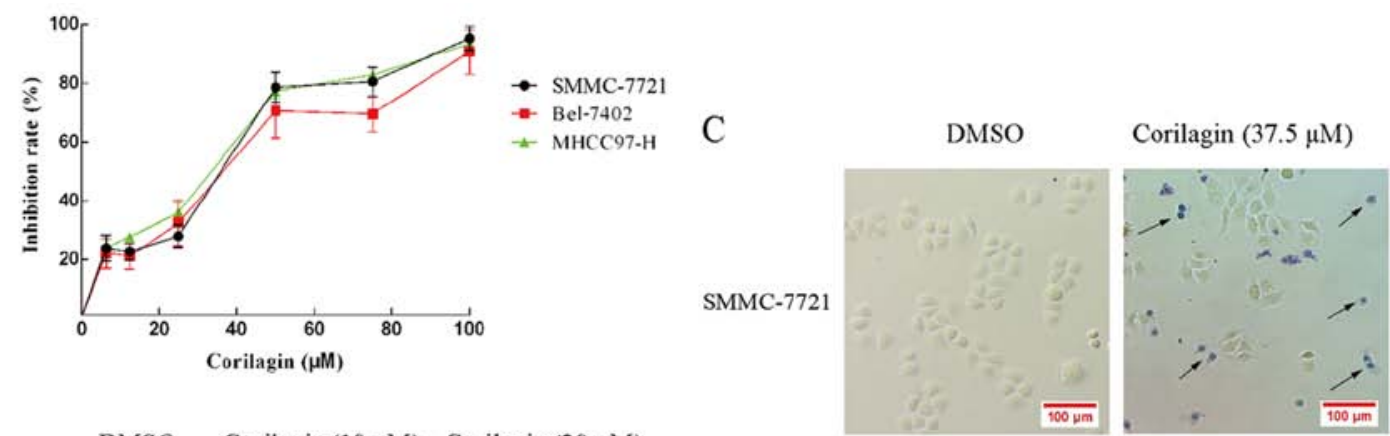

CDDP $(30 \mu \mathrm{M})$
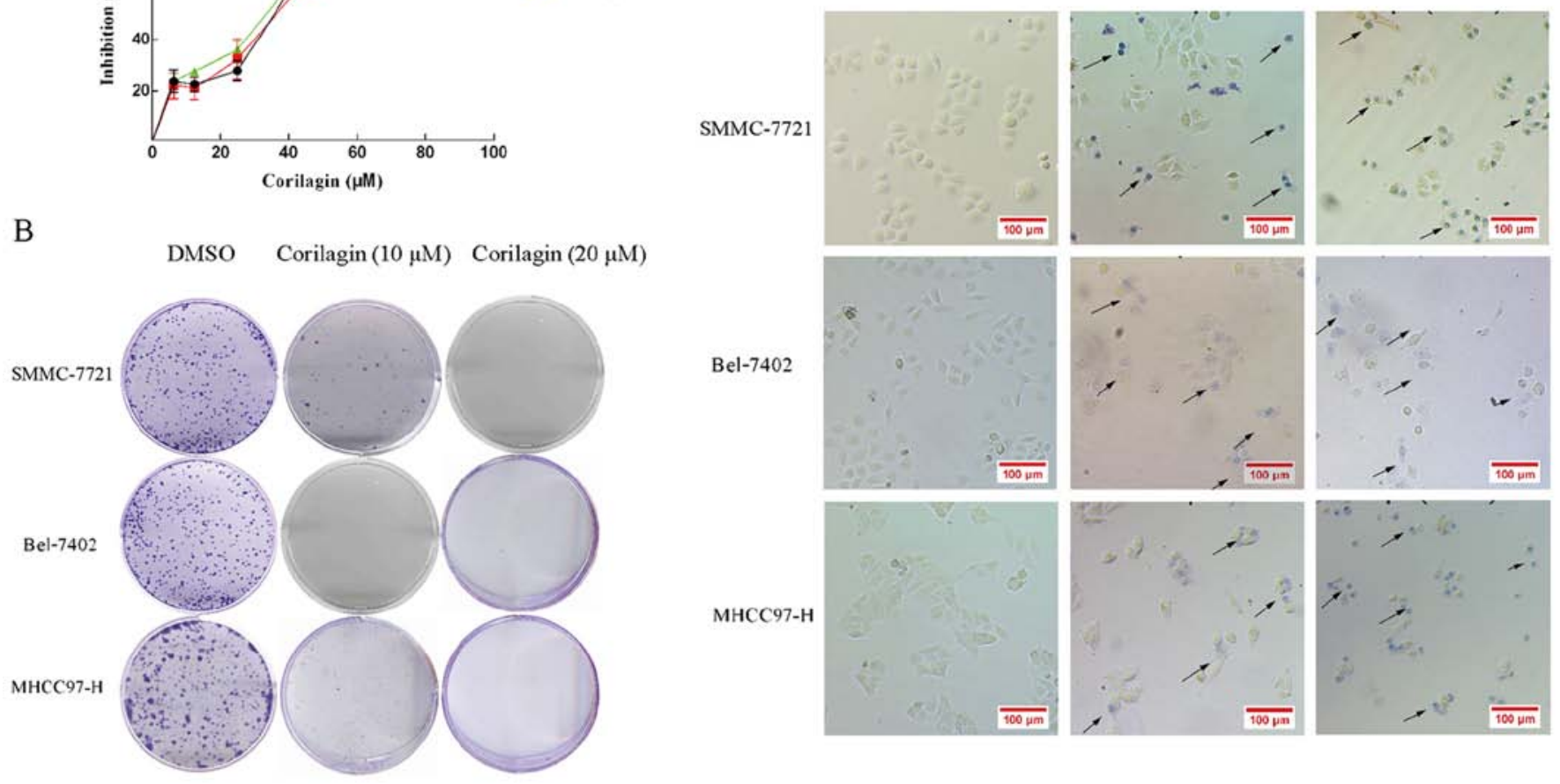

Figure 2. Growth inhibitory effect of corilagin on hepatocellular carcinoma (HCC) cells. (A) Three cell lines were treated with the indicated concentrations of corilagin for $24 \mathrm{~h}$, and cell survival was determined using the MTT assay and calculated as the percentage of the untreated control. (B) Analysis of colony formation capability of HCC cells treated with corilagin. (C) Dead cells were stained with trypan blue and present a blue color; arrows indicate dead cells. Magnification, x200.

visualized immediately using fluorescence microscopy (Leica Microsystems $\mathrm{GmbH}$ ).

Mitochondrial membrane potential assay. Cells were treated with $12.5,25,37.5$ and $50 \mu \mathrm{M}$ corilagin or DMSO $(0.1 \%$, w/v) as a negative control and $30 \mu \mathrm{M} C D D P$ as a positive control for $24 \mathrm{~h}$. The cells were then incubated with JC-1 $(0.1 \mu \mathrm{g} / \mathrm{ml})$ for $15 \mathrm{~min}$. After washing with PBS, the cells were suspended in PBS and observed with a fluorescence microscope as previously described (23). The green fluorescence from the JC-1 monomer and the red fluorescence from the aggregated form of JC-1 were visualized.

Flow cytometry assay. SMMC-7721 cells were seeded into $60-\mathrm{mm}$ plates $\left(1-2 \times 10^{5} /\right.$ plate $)$ and incubated with corilagin $(25$, 37.5 and $50 \mu \mathrm{M})$ or DMSO $(0.1 \%$, w/v) as a control on the next day. Control and treated cells were trypsinized after treatments for $24 \mathrm{~h}$. Cells were collected in PBS and stained using the Annexin V-FITC/PI Apoptosis Detection kit. The stained cells were analyzed by flow cytometry.

Western blot analyses. Cells were seeded into $60-\mathrm{mm}$ plates (1-2x10 $5 /$ plate) and incubated with corilagin $(12.5,25$ and $37.5 \mu \mathrm{M}$, which were below or approximately the $\mathrm{IC}_{50}$ ) or with DMSO $(0.1 \%, \mathrm{w} / \mathrm{v})$ as a control on the next day for $24 \mathrm{~h}$. Cells were washed in PBS, suspended in ice-cold lysis buffer containing $50 \mathrm{mM}$ Tris- $\mathrm{HCl}$ (pH 7.4), $5 \mathrm{mM}$ EDTA, $150 \mathrm{mM} \mathrm{NaCl}, 1 \%$ Triton X-100, $1 \%$ sodium deoxycholate, $1 \mathrm{mM}$ sodium, $20 \mathrm{mg} / \mathrm{ml}$ aprotinin, $20 \mathrm{mg} / \mathrm{ml}$ leupeptin and
$1 \mathrm{mM}$ phenylmethylsulfonyl fluoride (PMSF) and placed on ice for $5 \mathrm{~min}$. The solution was harvested then centrifugation was carried out at $12,000 \mathrm{x}$ g for $20 \mathrm{~min}\left(4^{\circ} \mathrm{C}\right)$, after which the supernatants were collected and the protein concentrations in the cell lysates were determined using the BCA assay. Whole cell lysates were resolved by SDS polyacrylamide gel electrophoresis and transferred onto nitrocellulose membranes, which were probed with anti- $\beta$-actin. Blots were then developed using ECL (Thermo Fisher Scientific, Inc.).

Statistical analysis. All data subjected to statistical analysis was from at least three independent experiments and are reported as the mean \pm standard deviation. The criterion for statistical significance was taken as $\mathrm{P}<0.05$ using a two-tailed t-test, and the count data were tested using Chi-square criterion comparing the frequency of parameters. The analyses were performed using SPSS 15.0 software (SPSS, Inc., Chicago, IL, USA).

\section{Results}

Corilagin inhibits the proliferation and colony formation of hepatocellular carcinoma cells. The cytotoxicity of corilagin in HCC cells was analyzed by the MTT assay. HCC cell lines, SMMC-7721, Bel-7402 and MHCC97-H, were treated with increasing concentrations of corilagin $(6.25,12.5,25,50,75$ and $100 \mu \mathrm{M})$ or with DMSO $(0.1 \%, \mathrm{w} / \mathrm{v})$ as a control for $24 \mathrm{~h}$. As shown in Fig. 2A, corilagin inhibited the proliferation of the three HCC cell lines and the inhibitory rate exhibited a dose-dependent trend. The $\mathrm{IC}_{50}$ values of corilagin against the 
A

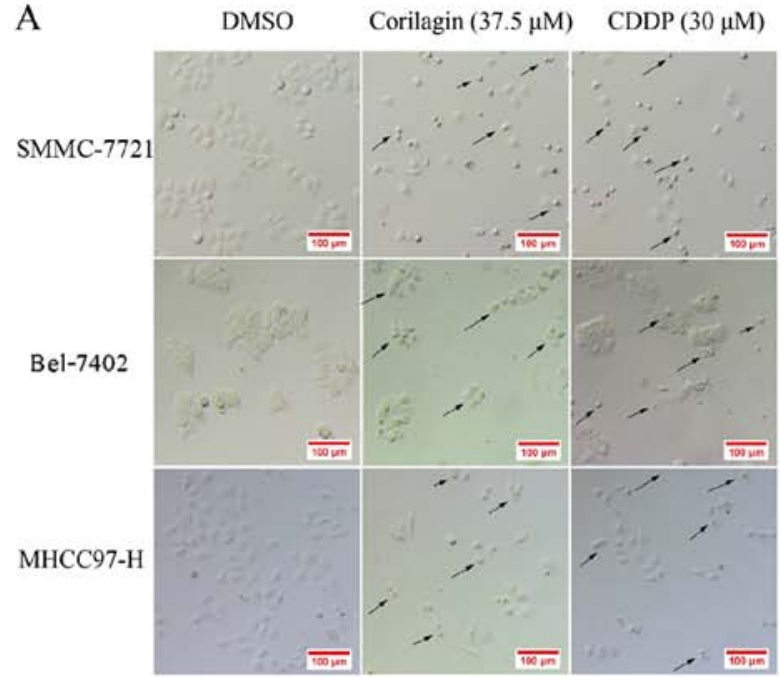

$\mathrm{C}$

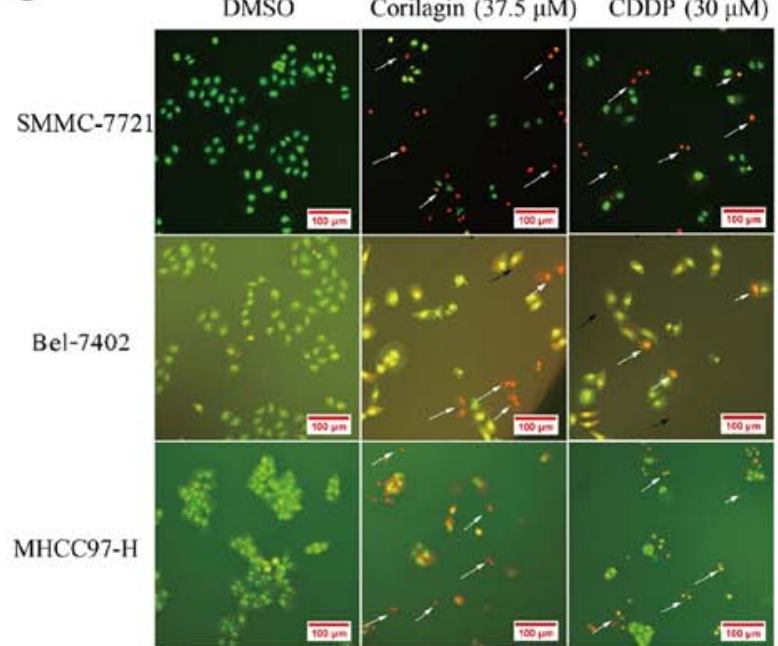

B

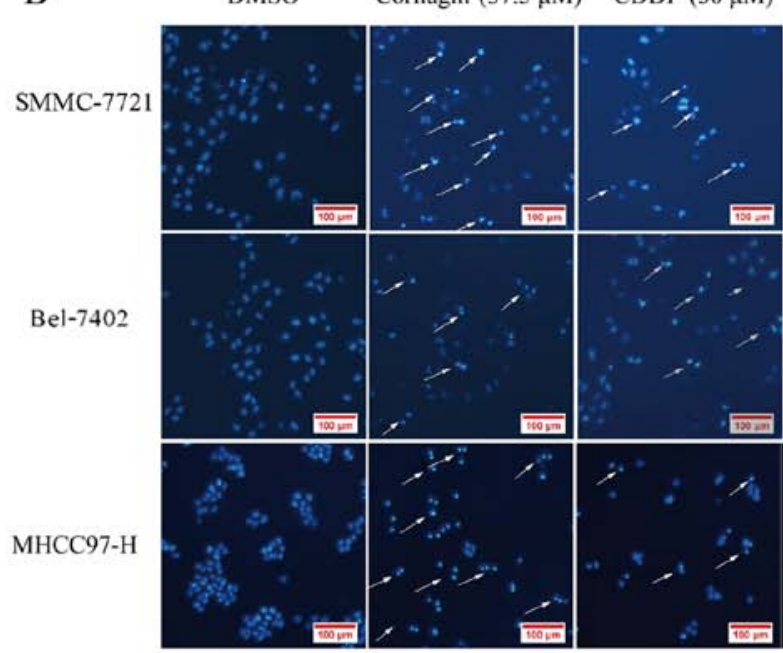

$\mathrm{D}$

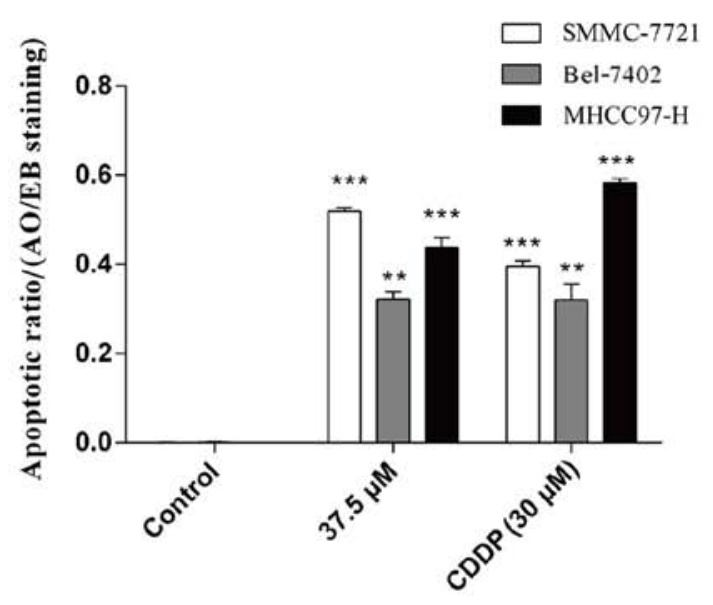

Figure 3. Growth inhibitory effect of corilagin on hepatocellular carcinoma (HCC) cells. SMMC-7721 cells were treated with DMSO $(0.1 \%$, w/v) or $37.5 \mu \mathrm{M}$ corilagin and CDDP as positive control for $24 \mathrm{~h}$. (A) Representative images after treatments. Images were captured using phase-contrast microscopy (Leica DM IRB); arrows indicate apoptotic cells. Magnification, x200. (B) Cells were fixed and stained using Hoechst 33258; apoptotic cells present brighter blue fluorescence; arrows indicate apoptotic cells. Magnification, x200. (C) Cells were harvested and stained with AO/EB; arrows indicate apoptotic cells. Magnification, $\mathrm{x} 200$. (D) The apoptotic ratio was determined in the AO/EB-stained apoptotic cells $\left({ }^{* *} \mathrm{P}<0.01\right.$ and $\left.{ }^{* * *} \mathrm{P}<0.001\right)$.

SMMC-7721, Bel-7402 and MHCC97-H cells were 38.12 \pm 1.2 $39.7 \pm 1.4$ and $37.05 \pm 0.9 \mu \mathrm{M}$, respectively. In the colony formation assay, corilagin showed a strong inhibitory effect on the HCC SMMC-7721 cells and HCC cell lines Bel-7402 and MHCC97-H (Fig. 2B). Corilagin presented s similar inhibitory rate on the three $\mathrm{HCC}$ cell lines. Based on the trypan blue staining assay (Fig. 2C), we observed trypan blue-stained cells in the corilagin-treated group indicating that corilagin can lead to the death of HCC SMMC-7721, Bel-7402 and MHCC97-H cells. These results showed that corilagin significantly suppressed the proliferation of the HCC cells.

Observation of the morphological changes in HCC cells after exposure to corilagin. Cell morphological changes after corilagin exposure were observed under a microscope. As shown in Fig. 3A, cells treated with corilagin for $24 \mathrm{~h}$ showed typical features of apoptotic cells which were similar to the features exhibited by the inhibitory effect of CDDP on the
HCC cells, such as nuclear fragmentation, cell shrinkage and apoptotic bodies under the microscope. In addition, based on the Hoechst 33258 staining assay, we found that the blue emission light in apoptotic cells was much brighter than that in the control cells (Fig. 3B). Upon AO/EB staining assay, different cells (alive, apoptotic or necrotic) were clearly differentiated by the different colors (Fig. 3C). Fig. 3D shows the quantification of 20 different images and indicates the apoptotic ratios between the control and corilagin-treated cells. These results indicated that the corilagin-treated cells exhibited morphological changes indicating apoptosis, including chromatin condensation and nuclear fragmentation.

Effect of corilagin on the apoptosis of HCC cells. To determine the apoptotic effect of corilagin on HCC cells, SMMC-7721 cells were treated with different concentrations of corilagin $(25,37.5$ and $50 \mu \mathrm{M})$ for $24 \mathrm{~h}$. Cells were evaluated by flow cytometry through Annexin V/PI staining. As shown in Fig. 4, 


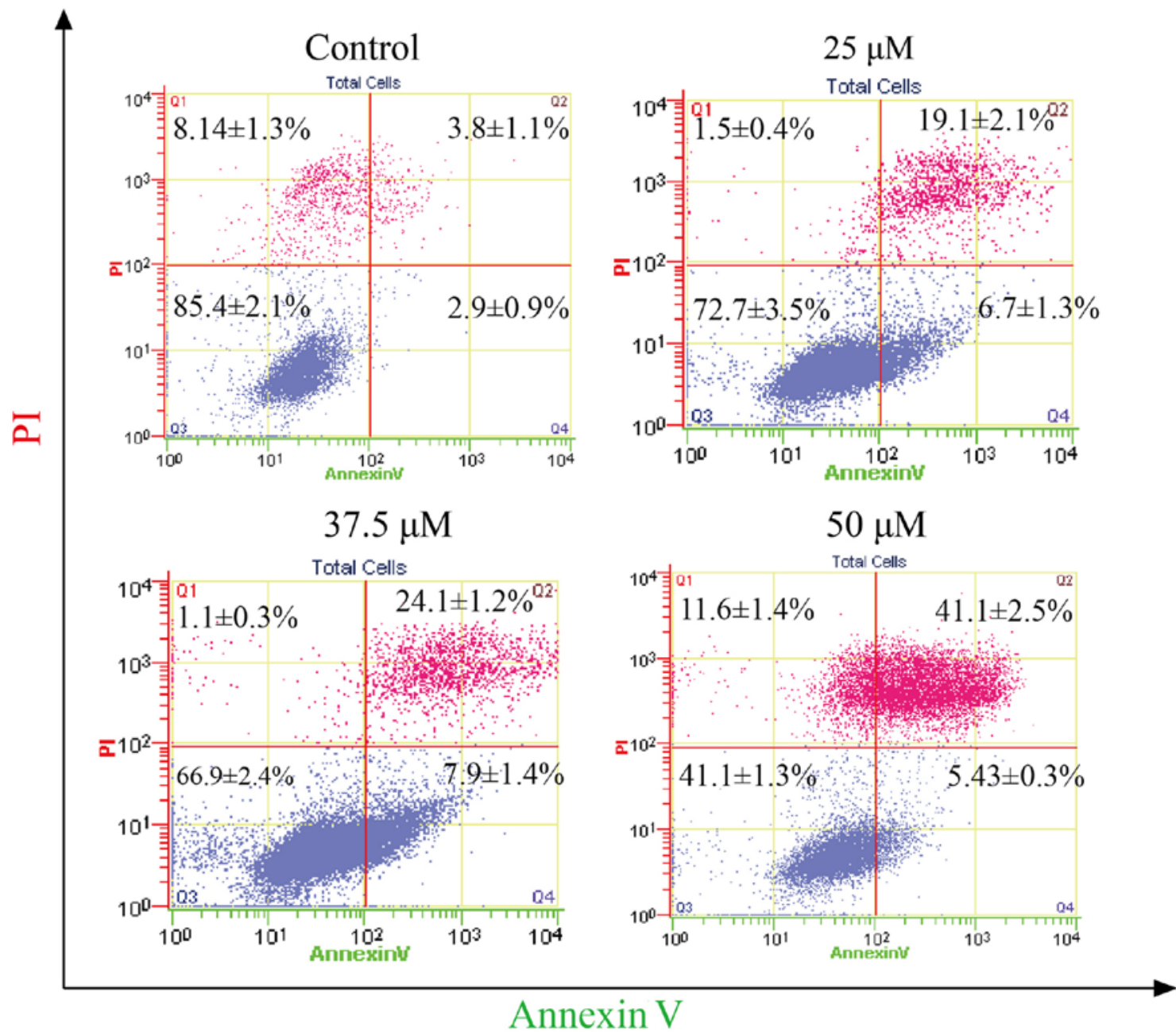

Figure 4. Effect of corilagin on the percentage of apoptotic hepatocellular carcinoma (HCC) cells. SMMC-7721 cells were treated with different doses $(25,37.5$ and $50 \mu \mathrm{M})$ of corilagin for $24 \mathrm{~h}$ and then stained with Annexin V/PI staining and then evaluated by flow cytometry.

A
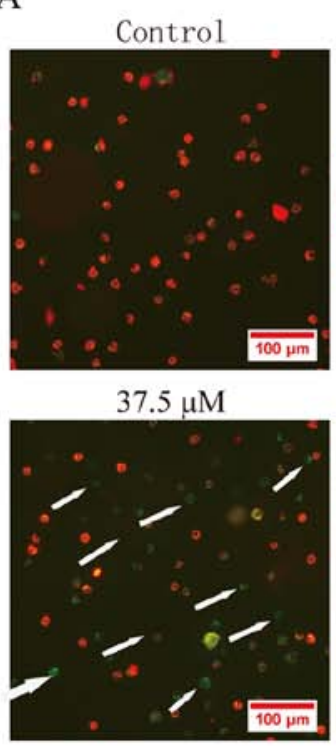

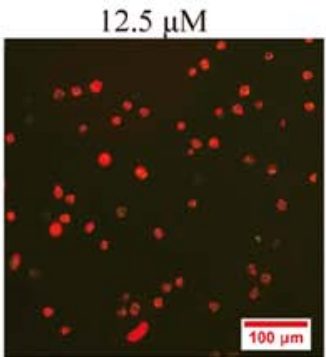

$50 \mu \mathrm{M}$

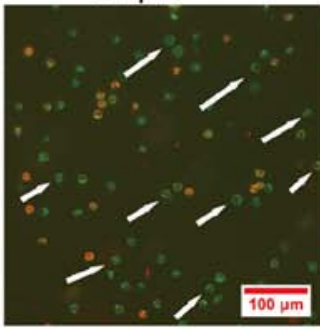

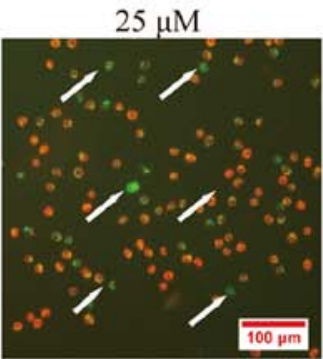

$30 \mu \mathrm{M}(\mathrm{CDDP})$

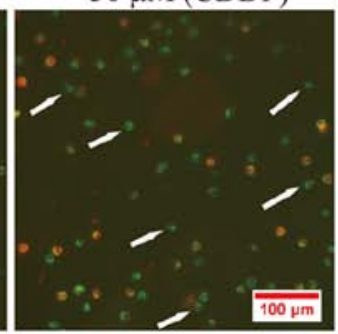

$\mathrm{B}$

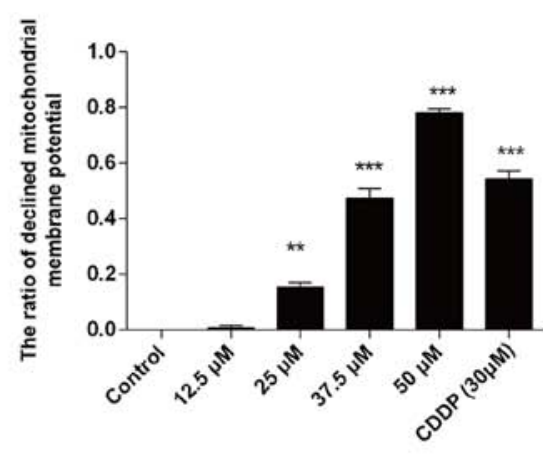

$\mathrm{C}$

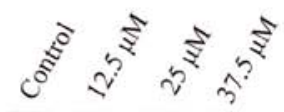

\section{Cyto c}

Tubulin

Figure 5. Corilagin treatment reduces the mitochondrial transmembrane potential of hepatocellular carcinoma (HCC) SMMC-7721 cells. (A) Cells were treated with different doses $(12.5,25,37.5$ and $50 \mu \mathrm{M})$ of corilagin or DMSO $(0.1 \%$, w/v) as a negative control and $30 \mu \mathrm{M}$ CDDP as a positive control for $24 \mathrm{~h}$ and then stained with JC-1. Images were captured by fluorescence microscopy. Magnification, $\mathrm{x} 100$. (B) The ratio of the decrease in mitochondrial transmembrane potential in SMMC-7721 cells treated with corilagin $\left({ }^{* *} \mathrm{P}<0.01\right.$ and $\left.{ }^{* * * *} \mathrm{P}<0.001\right)$. (C) The protein level of cytochrome $c$ (Cyto c) in the cytoplasm was increased following the treatment of corilagin as determined by western blot analysis. 
A

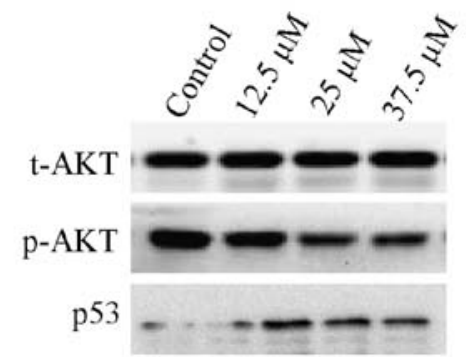

Bcl-2

Survivin

Caspase- 9

Cleaved-caspase-9

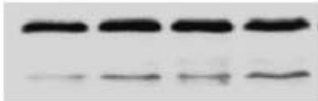

Cleaved-caspase- 3

Caspase-3
-caspase-3

$$
\begin{array}{r}
\text { PARP } \\
\text { Cleaved-PARP }
\end{array}
$$

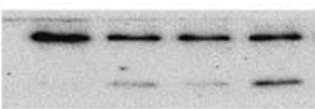

Tubulin
$\mathrm{B}$

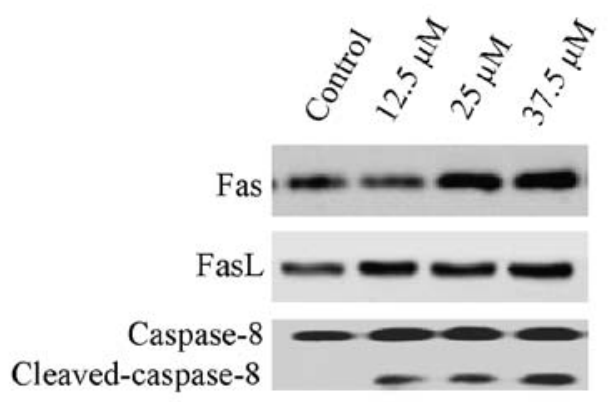

Tubulin

Figure 6. Expression of apoptosis-related proteins in SMMC-7721 cells following treatment with different concentrations of corilagin $(0,12.5,25$ and $37.5 \mu \mathrm{M})$ for $24 \mathrm{~h}$. (A) p-AKT, Bcl-2 and survivin were downregulated, p53 was upregulated, and caspase-9, caspase-3 and PARP were cleaved following corilagin treatment. (B) Fas and FasL were upregulated and caspase-8 was cleaved following corilagin treatment.

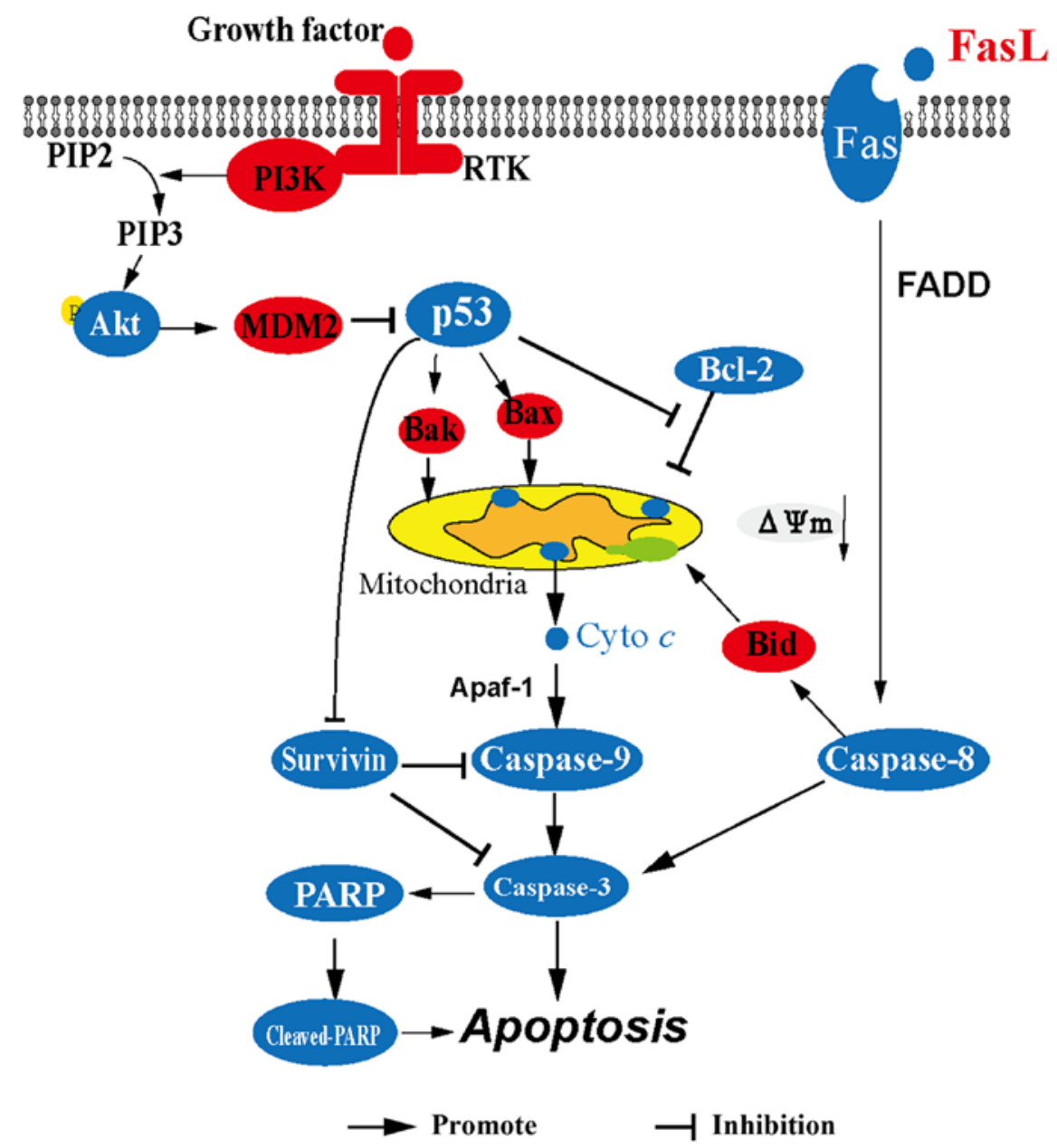

Figure 7. The signaling pathway which is involved in the antitumor effects of corilagin. 
increasing percentages of apoptotic cells were observed when compared to the control group. The apoptotic percentage of the HCC cells was $41.1 \%$ following treatment with corilagin of $50 \mu \mathrm{M}$.

Effect of corilagin on the mitochondrial transmembrane potential of HCC cells. To investigate the loss of $\Delta \psi \mathrm{m}$ during apoptosis induced by corilagin, cells were treated with 12.5 , $25,37.5$ and $50 \mu \mathrm{M}$ corilagin or DMSO as a negative control and $30 \mu \mathrm{M}$ CDDP as a positive control for $24 \mathrm{~h}$ and then stained with JC-1 and monitored with a fluorescence microscope. JC-1 forms monomer and emits green fluorescence when $\Delta \psi \mathrm{m}$ is depolarized (common in apoptosis), while JC-1 aggregates and emits red fluorescence at a highly polarized $\Delta \psi \mathrm{m}$. As shown in Fig. 5A, JC-1 was accumulated in the control cells which presented red fluorescence indicating a high $\Delta \psi \mathrm{m}$. However, JC-1 was poorly accumulated in the corilagin-treated cells, which displayed only green or weak red fluorescence, indicating low $\Delta \psi \mathrm{m}$ in the mitochondria of the corilagin-treated cells. Fig. 5B shows the quantification of the ratio of decreased mitochondrial membrane potential in the cells treated with corilagin. Moreover, Fig. 5C shows that the protein level of cytochrome $c$ (Cyto c) in the cytoplasm was increased following the treatment of corilagin which can lead to the activation of caspase-9 and caspase-3. This result indicates activation of the intrinsic apoptotic signaling pathways.

Effect of corilagin on the levels of apoptosis-related proteins in HCC cells. Apoptosis is often relevant to a well-organized sequence of cellularevents, resulting in the alteration of intracellular signaling pathways. HCC cells were treated with different concentrations of corilagin $(0,12.5,25$ and $37.5 \mu \mathrm{M})$ for $24 \mathrm{~h}$, and cytochrome $c$ was translocated from the mitochondria to the cytoplasm which confirmed the result of the mitochondrial membrane potential assay. As shown in Fig. 6A, the expression of p-AKT was downregulated, while the p53 protein level was upregulated following treatment with corilagin. Cleavage of caspase-9, caspase-3 and PARP was observed, indicating activation of the intrinsic apoptotic signaling pathways (Fig. 6A). In addition, upregulation of Fas and FasL was noted which led to the activation of caspase-8 (Fig. 6B); caspase-8 is an important protein of the extrinsic apoptotic signaling pathways, namely the Fas/FasL signaling pathway. These results indicate that corilagin also activates the extrinsic signaling pathways. We also observed downregulation of Bcl-2 and survivin which are important anti-apoptotic proteins and may facilitate the inhibitory activity of corilagin on HCC cells (Fig. 6A).

\section{Discussion}

Corilagin is the main bioactive component of many traditional herbal plants (e.g., P. niruri L. and P. emblica L.). Corilagin was found to inhibit the growth of many types of cancer cells including nasopharyngeal carcinoma (19), HCC (2), gastric adenocarcinoma (19), lung adenocarcinoma (20), metrocarcinoma (18), colon cancer (19), ovarian cancer (3) and osteosarcoma cells (3) of human origin and murine melanoma cells (18).

In a previous study, our group found that corilagin inhibited the growth of HCC cells by inducing G2/M phase arrest by downregulating p-Akt and cyclin B1/cdc2 and upregulating p-p53 and p21 (2) and corilagin inhibited ovarian cancer cells via the TGF- $\beta$ /Akt/ERK signaling pathways (3). Moreover, corilagin was reported to enhance the antitumor activity of CDDP in HCC cells (21). In the present study, we found that corilagin-treated cells exhibited morphological changes indicative of apoptosis, including chromatin condensation and nuclear fragmentation through Hoechst 33258 and AO/EB staining assays demonstrating that corilagin can induce the apoptosis of HCC cells. The result of the flow cytometry assay was in accordance with the observation of morphological change; corilagin does induce the apoptosis of HCC cells.

At the molecular level, we also observed a change in the mitochondrial membrane potential in the corilagin-treated HCC cells. This result confirmed the work of Wang who found that corilagin induced membrane potential change in the mitochondria of human gastric cancer SGC-7901 cells (12). In the present study, cytochrome $c$ was translocated from mitochondria to the cytoplasm which confirmed the result of the mitochondrial membrane potential assay. Cleavage of caspase-9, caspase-3 and PARP was observed demonstrating that corilagin can activate the intrinsic apoptotic signaling pathways, namely the mitochondrial pathway. In the western blot assay of corilagin-treated HCC cells, we observed activation of caspase-8, which is downstream of the death receptor pathway (Fas/FasL signaling pathway) and upregulation of Fas/FasL (Fig. 6B). Activation of caspase- 8 indicated that corilagin can also activate the death receptor pathway. In addition, we also observed downregulation of Bcl-2 and survivin and upregulation of p53. Bcl-2 is an important antiapoptotic protein which can block the release of cytochrome $c$ from mitochondria to the cytoplasm (24). Survivin is also an important anti-apoptotic protein which can inhibit the activity of caspase-3 (25). p53 is an important pro-apoptotic protein which can promote the release of cytochrome $c$ and activation of the intrinsic apoptotic signaling pathways (26).

In summary (Fig. 7), corilagin upregulates the expression of p53 leading to the release of cytochrome $c$ from the mitochondria to the cytoplasm and then cytochrome $c$ activates caspase-9, caspase-3 and cleavage of PARP consequently resulting in the apoptosis of HCC cells. In addition, corilagin activates the Fas/FasL/caspase- 8 signaling pathway which can lead to the activation of caspase- 3 and the cleavage of Bid which contributes to the release of cytochrome $c$. Downregulation of survivin also facilitates the activity of caspase-9 and caspase-3. These results suggest that corilagin significantly induces the apoptosis of HCC cells through both the mitochondrial apoptotic pathway and the death receptor pathway. Our findings are beneficial to the ongoing research on corilagin, and provide evidence for the potential use of corilagin as an antitumor drug.

\section{Acknowledgements}

Not applicable.

\section{Funding}

This study was supported by grants from the National Natural Science Foundation of China (grant no. 81274149), the Xiamen 
Municipal Science and Technology Innovation Fund Project of China (grant no. 3502Z20130038), the Construction Projects of the Top University at Fujian Agriculture and Forestry University of China (grant no. 612014042), and the Science and Technology Plan Projects of Xiamen (grant no. 3502Z20142003).

\section{Availability of data and materials}

The datasets used during the present study are available from the corresponding author upon reasonable request.

\section{Authors' contributions}

YM conceived and designed the experiments; YD, XL, XL, $\mathrm{ZZ}, \mathrm{WH}$ and LC performed the experiments; YD and YM analyzed the data and wrote the paper; YM and QT revised the paper.

\section{Ethics approval and consent to participate}

Not applicable.

\section{Consent for publication}

Not applicable.

\section{Competing interests}

The authors declare that they have no competing interests.

\section{References}

1. Singh S, Singh PP, Roberts LR and Sanchez W: Chemopreventive strategies in hepatocellular carcinoma. Nat Rev Gastroenterol Hepatol 11: 45-54, 2014.

2. Ming Y, Zheng Z, Chen L, Zheng G, Liu S, Yu Y and Tong Q: Corilagin inhibits hepatocellular carcinoma cell proliferation by inducing G2/M phase arrest. Cell Biol Int 37: 1046-1054, 2013.

3. Jia L, Jin H, Zhou J, Chen L, Lu Y, Ming Y and Yu Y: A potential anti-tumor herbal medicine, Corilagin, inhibits ovarian cancer cell growth through blocking the TGF- $\beta$ signaling pathways. BMC Complement Altern Med 13: 33, 2013.

4. Schmidt OT and Lademann R: Corilagin, ein weiterer kristallisierter Gerbstoff aus Dividivi. X. Mitteilung über natürliche Gerbstoffe. Justus Liebigs Ann Chem 571: 232-237, 1951 (In German).

5. Pham AT, Malterud KE, Paulsen BS, Diallo D and Wangensteen H: DPPH radical scavenging and xanthine oxidase inhibitory activity of Terminalia macroptera leaves. Nat Prod Commun 6: 1125-1128, 2011.

6. Yang F, Wang Y, Xue J, Ma Q,Zhang J, Chen YF, Shang ZZ,Li QQ, Zhang SL and Zhao L: Effect of Corilagin on the miR-21/smad7/ ERK signaling pathway in a schistosomiasis-induced hepatic fibrosis mouse model. Parasitol Int 65: 308-315, 2016.

7. Dong XR, Luo M, Fan L, Zhang T, Liu L, Dong JH and Wu G: Corilagin inhibits the double strand break-triggered NF-kappaB pathway in irradiated microglial cells. Int J Mol Med 25: 531-536, 2010.
8. Park JH, Joo HS, Yoo KY, Shin BN, Kim IH, Lee CH, Choi JH, Byun K, Lee B, Lim SS, et al: Extract from Terminalia chebula seeds protect against experimental ischemic neuronal damage via maintaining SODs and BDNF levels. Neurochem Res 36: 2043-2050, 2011

9. Duan W, Yu Y and Zhang L: Antiatherogenic effects of phyllanthus emblica associated with corilagin and its analogue. Yakugaku Zasshi 125: 587-591, 2005.

10. Yang MH, Vasquez Y, Ali Z, Khan IA and Khan SI: Constituents from Terminalia species increase PPAR $\alpha$ and PPAR $\gamma$ levels and stimulate glucose uptake without enhancing adipocyte differentiation. J Ethnopharmacol 149: 490-498, 2013.

11. Gambari R, Hau DK, Wong WY and Chui CH: Sensitization of Hep3B hepatoma cells to cisplatin and doxorubicin by corilagin. Phytother Res 28: 781-783, 2014.

12. Wang BQ: Corilagin nanoparticle-induced apoptosis in human gastric cancer SGC-7901 cells via the mitochondrial pathway. Acta Pharmacol Sin 34: 14-14, 2013.

13. Kakiuchi N, Hattori M, Namba T, Nishizawa M, Yamagishi T and Okuda T: Inhibitory effect of tannins on reverse transcriptase from RNA tumor virus. J Nat Prod 48: 614-621, 1985.

14. Berry DE, MacKenzie L, Shultis EA, Chan JA and Hecht SM: Naturally occurring inhibitors of topoisomerase I mediated DNA relaxation. J Org Chem 57: 420-422, 1992.

15. Hecht SM, Berry DE, MacKenzie LJ, Busby RW and Nasuti CA: A strategy for identifying novel, mechanistically unique inhibitors of topoisomerase I. J Nat Prod 55: 401-413, 1992.

16. Komori A, Yatsunami J, Suganuma M, Okabe S, Abe S, Sakai A, Sasaki K and Fujiki H: Tumor necrosis factor acts as a tumor promoter in BALB/3T3 cell transformation. Cancer Res 53: 1982-1985, 1993.

17. Okabe S, Suganuma M, Imayoshi Y, Taniguchi S, Yoshida T and Fujiki $\mathrm{H}$ : New TNF-alpha releasing inhibitors, geraniin and corilagin, in leaves of Acer nikoense, Megusurino-ki. Biol Pharm Bull 24: 1145-1148, 2001.

18. Zhang YJ, Nagao T, Tanaka T, Yang CR, Okabe H and Kouno I: Antiproliferative activity of the main constituents from Phyllanthus emblica. Biol Pharm Bull 27: 251-255, 2004.

19. Liu Z, Wang D, Chen Y, Ren L, Li K and Zhang W: Experiment studies on the pharmacodynamics experiment by corilagin. Zhongliu Fangzhi Yanjiu 29: 356-358, 2002.

20. Chen Y and Ren L: Studies on the anti-cancer active constituents of matsumura leafflower (Phyllanthus matsumarae) II. Isolation and identification of polyphenolic compounds. Chin Tradit Herbal Drugs 28: 198-202, 1997.

21. Zheng ZZ, Chen LH, Liu SS, Deng Y, Zheng GH, Gu Y and Ming YL: Bioguided fraction and isolation of the antitumor components from Phyllanthus niruri L. Biomed Res Int 2016: 9729275, 2016.

22. Jia L, Zhou J, Zhao H, Jin H, Lv M, Zhao N, Zheng Z, Lu Y, Ming Y and Yu Y: Corilagin sensitizes epithelial ovarian cancer to chemotherapy by inhibiting Snail glycolysis pathways. Oncol Rep 38: 2464-2470, 2017.

23. Chazotte B: Labeling mitochondria with JC-1. Cold Spring Harb Protoc: Sep 1,2011 (Epubahead of print). doi: 10.1101/pdb.prot065490.

24. Lampson BL and Davids MS: The development and current use of BCL-2 inhibitors for the treatment of chronic lymphocytic leukemia. Curr Hematol Malig Rep 12: 11-19, 2017.

25. Shamsabadi FT, Eidgahi MR, Mehrbod P, Daneshvar N, Allaudin ZN, Yamchi A and Shahbazi M: Survivin, a promising gene for targeted cancer treatment. Asian Pac J Cancer Prev 17: 3711-3719, 2016.

26. Coutts AS, Adams CJ and La Thangue NB: p53 ubiquitination by Mdm2: A never ending tail? DNA Repair (Amst) 8: 483-490, 2009. 